\title{
Retrolental Fibroplasia: Evidence for a Role of the Prostaglandin Cascade in the Pathogenesis of Oxygen-Induced Retinopathy in the Newborn Beagle
}

\author{
ROBERT W. FLOWER ${ }^{(27)}$ AND DAVID A. BLAKE WITH THE ASSISTANCE OF \\ STEPHEN D. WAJER, PATRICIA G. EGNER, D. SCOTT MCLEOD, AND SHARON M. PITTS \\ Applied Physics Laboratory [R. W. F.], The Wilmer Ophthalmological Institute [R. W. F., S. D. W., D. S. M., \\ S. M. P.], The Department of Pharmacology [D. A. B.], and The Department of Gynecology and Obstetrics [D. A. B., \\ P. G. E.I of The Johns Hopkins University School of Medicine and Hospital, Baltimore, Maryland, USA
}

\begin{abstract}
Summary
Aspirin administration, at a dosage producing plasma levels within the human therepeutic range, caused marked inhibition of production of both vascular prostacyclin (a vasodilator) and platelet thromboxane (a vasoconstrictor) in beagle puppies. In addition, aspirin-treated, oxygen-exposed puppies developed retinopathy of significantly greater severity than their unmedicated, oxygen-exposed littermates. Direct ophthalmoscopic observations indicated that whereas sustained oxygen breathing produced retinal vasoconstriction in unmedicated puppies, retinal vessels of aspirintreated littermates became more dilated or remained unchanged. It is postulated that retinal vasoconstriction may be a normal physiologic mechanism to protect the immature retina from damaging effects of high blood oxygen levels; i.e., it may be a protective rather than a pathologic process in response to hyperoxia.

Many vascular anomalies which characterize the human disease were present in the retinas of the puppies. Several of the most severely affected puppies treated with aspirin even displayed grade III cicatricial retinopathy (falciform retinal fold). Thus, a major criticism of the retrolental fibroplasia animal model has been addressed by producing cicatricial retrolental fibroplasia in puppies, and the confidence with which results from experimental animal studies might be extrapolated to the clinical situation is thereby strengthened.
\end{abstract}

\section{Speculation}

It is conceivable that the susceptibility of any eye to oxygenassociated retinopathy at birth depends upon the extent to which the vasoconstriction protective response is functional as well as upon the degree of retinal vascular maturity attained. In utero, the arterial $\mathrm{Po}_{2}$ is low, and blood flow to the immature retina is high. When the $\mathrm{PO}_{2}$ rises at birth, retinal blood flow does not change, although arterial blood pressure rises; this could be explained by the occurrence of some degree of vasoconstriction in response to elevated arterial $\mathrm{Po}_{2}$ or reduced $\mathrm{PCO}_{2}$ which establishes the clinically normal retinal vasotonia. In light of this, those cases of "spontaneous" retrolental fibroplasia reported in premature infants never administered oxygen or in full-term infants who were administered oxygen might simply be examples of individuals with inadequate retinal vasotonia for protection of structurally immature vessels from the effects of elevated arterial $\mathbf{P O}_{2}$ and/or excessively high transmural pressure.

Retrolental fibroplasia (RLF), first identified by Terry in 1942 (24), is today often referred to more properly as the retinopathy of prematurity. Occurrence of this retinopathy has since been firmly linked to hyperoxia (18). From clinical observations, onset of oxygen-associated retinopathy appears to consist of two distinct stages. The primary stage, occurring during prolonged breathing of high concentrations of oxygen, involves closure of the immature retinal vasculature. Normal vascular growth proceeding from the optic disk toward the retinal periphery is then halted as long as the blood vessels remain occluded. During the secondary stage, which occurs upon return to ambient air breathing, retinal vessels not permanently occluded can undergo neovascular proliferation. These new vessels may lack normal structural integrity and be prone to hemorrhage. Progression of the retinopathy beyond the second stage is nonspecific and depends on the degree of hemorrhage and subsequent retinal traction which may occur.

Much of the basic research on these specific stages of vascular changes has been done using experimental animal models. The dog, cat, and rat at full-term birth have retinal vasculatures comparable in terms of maturity to that of the human fetus at about $61 / 2$ months' gestation. Of these animal models, investigators have predominantly used the kitten. A major criticism of this model is the inability to produce the cicatricial form of retinopathy which occurs in human eyes. Inasmuch as direct study of newborn human retinal physiology and histology is virtually prohibitive, the animal model plays a role of considerable importance.

Results of studies on the young kitten eye (2) indicated selective destruction of endothelial tissue during RLF vascular degenerative processes; all other cells in the vicinity of affected capillaries were normal. This suggests that vessel degeneration resulted from cytotoxic effects of oxygen on endothelial cells rather than from secondary effects due to vasoconstriction and diminished blood flow. Further, these data precluded the possibility of either establishing the cause of vascular damage or conclusively identifying the mechanism of oxygen vaso-obliteration. These questions have thus remained without definitive answers.

Recent evidence has linked manipulation of the prostaglandin system to oxygen-dependent changes in the ductus arteriosus $(6$, 12). This led us to look for a similar link between the prostaglandin system and oxygen-induced changes in the immature retinal vasculature. Hence, the present study was undertaken with two goals: first, to demonstrate and standardize an improved RLF animal model by administering oxygen to purebred beagle puppies in precisely controlled environments, and second, using this model, to elucidate the possible role of prostaglandin biosynthesis on RLF pathogenesis. The effect of aspirin on production of RLF in the puppy was investigated because this drug has been shown to inhibit conversion of arachidonic acid to a potent vasodilator (prostacyclin) by endothelial cells (25) and a potent vasoconstrictor (thromboxane) by platelets (21). 
METHODS AND RESULTS

\section{ASPIRIN EFFECTS ON RLF PRODUCED BY CHRONIC OXYGEN} EXPOSURE

Methods. Four litters, each containing five to seven full-term beagle puppies (64 to 67 days gestation), were used in this phase of the study. At 2 days of age, the puppies from each litter were randomly allocated to receive either an aqueous suspension of 20 $\mathrm{mg}$ aspirin in a $1 \mathrm{ml}$ water per os or $1 \mathrm{ml}$ of water. The aspirin dose (St. Joseph's Baby Aspirin tablets, $1.25 \mathrm{~g}$ aspirin per tablet, were used) was expected to produce circulating salicylate levels in the range of that found in clinical therapeutics. The puppies and their mothers were then placed in an air-tight chamber ventilated with humidified 100\% oxygen. The puppies were kept in the chamber continuously for $72 \mathrm{hr}$ and were administered aspirin in water or water only every $24 \mathrm{hr}$, and the mother was introduced into and removed from the chamber through an air lock to nurse the puppies for one 6-hr period each day. Throughout exposure, chamber oxygen content was held between 95 and $100 \%$; drops of less than $10 \%$ were experienced over one-half $\mathrm{hr}$ periods when introducing or removing the mother. During exposure of the first litter, chamber $\mathrm{CO}_{2}$ was monitored but not controlled, and $\mathrm{CO}_{2}$ levels reached as high as $2 \%$ with puppies in the chamber and $4 \%$ when the nursing mother was also present. However, during exposure of the other three litters, chamber $\mathrm{CO}_{2}$ content was held constant at a level close to that in room air $(0.033 \%)$ by a higher oxygen flow rate and by placing containers of soda lime chips in the chamber.

Fourteen days after completion of oxygen exposure, each puppy was anesthetized with sodium pentobarbital and maintained on a respirator (Bourns LS-104), and color fundus photographs of each eye were made. Both carotid arteries were then progradedly cannulated, the puppy was heparinized, both jugular veins were severed, and approximately $100 \mathrm{ml}$ of India ink were slowly infused and circulated through the head for $5 \mathrm{~min}$. Both eyes were enucleated, and whole retina flat mounts were prepared. The India ink retinal flat mounts of both eyes from each puppy were evaluated for pathologic changes according to a standard scoring system (20) for severity of RLF by four evaluators who were unaware of the source of each preparation. Rating scores were statistically analyzed for significant differences by a four-way analysis of variance examining the effect of treatment, left versus right eye, evaluators, and litters.

To determine the effect of aspirin alone on the retinal vasculature, a fifth litter of four puppies was treated in an identical manner (three were given aspirin in water and one was given only water), except it was maintained in room air the entire time. As with the previous litters, the mother was permitted to nurse only once a day.

Results. In Table 1, severity scores are reported for each litter by treatment group weighted for litter effect. There was, however, no significant effect introduced on the basis of left versus right eye or by the evaluators. The data demonstrate that aspirin-treated, oxygen-exposed puppies presented with retinopathy of significantly greater severity than their unmedicated, oxygen-exposed littermates, although the degree of retinal pathology differed somewhat among litters. It is of interest that the litter with the highest scores (litter 1) experienced elevated carbon dioxide levels during oxygen administration, whereas the three subsequent experiments were modified to keep the carbon dioxide at a minimal level. None of the puppies from the fifth litter which received aspirin and no oxygen showed any retinopathy.

The retinal flat mount preparations of six pairs of puppy eyes from litter 4 are shown in Figure 1. The eyes from a seventh littermate which was in the aspirin-treated group are not included here; for unexplained reasons, it presented with pathology no more severe than that of the unmedicated, or placebo, group. Otherwise, this litter presented the most marked distinction between aspirin-treated and unmedicated littermates yet seen. Pre-
Table 1. Effect of aspirin on oxygen-induced RLF in newborn beagles $^{1}$

\begin{tabular}{cccc}
\hline & \multicolumn{2}{c}{ Severity score $^{2}$} & $\begin{array}{c}\text { Aspirin- } \\
\text { associated } \\
\text { change }\end{array}$ \\
\cline { 2 - 4 } Litter & Control & Aspirin-treated & +2.1 \\
\hline 1 & $7.3 \pm 1.1^{3}(3)^{4}$ & $9.4 \pm 1.2(2)$ & +1.3 \\
2 & $4.5 \pm 1.1(3)$ & $5.8 \pm 1.1(3)$ & +1.6 \\
3 & $4.1 \pm 1.2(2)$ & $5.7 \pm 1.0(4)$ & +2.5 \\
4 & $5.8 \pm 1.1(3)$ & $8.3 \pm 1.0(4)$ & \\
\hline
\end{tabular}

${ }^{1}$ Two day-old puppies were maintained for 3 days in 95 to $100 \%$ oxygen. Aspirin was administered orally to one-half of the littermates for 5 days at a daily dose of $20 \mathrm{mg}$; the first dose was given just before placing the animals in oxygen.

${ }^{2}$ Ratings of India ink flat mounts of retinas prepared from 3-wk-old puppies. Four raters used a standard scoring system (20) which indexed severity of pathology within a range of 0 to 12 . Treatment effect is significant $(P<0.0001)$ by analysis of variance.

${ }^{3}$ Mean \pm S.E. weighted for litter effect.

${ }^{4}$ Numbers in parentheses, number of puppies per treatment group (two eyes from each puppy were scored).

sentation of the data in this format lead to some interesting observations. In these eyes, contrary to previously reported observations in animal RLF, the most severe pathology appeared in the nasal rather than in the temporal retina. It may also be seen that a dorsal lobe of vessels formed in the aspirin-treated eyes consisted largely of what appear to be arteriovenous shunts; some evidence of these shunt-like vessels are also present in the placebo group eyes.

In several of the most severely affected puppies, grade III cicatricial retinopathy (falciform retinal fold) was found. Figure 2 shows composite fundus photographs of both eyes and a retinal flat mount of one of these puppies. Significant venous distention as well as evidence of hemorrhage was common in the eyes of these puppies. Moreover, examination of the retinal flat mount preparations and fluorescein angiograms revealed examples of many of the vascular anomalies which characterize the human disease $(10,15)$. In Figure 3, "nubbins" of tissue identified as anastomoses of venules and arterioles in regressing human RLF $(A)$ are compared to similar structures found in the puppy retina $(B)$, and the "wishbone" type of arteriovenous anastomoses also found in human RLF ( $C$, fluorescein) are compared to similar structures in the periphery of the puppy retina ( $D$, arrows). Likewise in Figure 4, the sharp border between vascularized and avascular retina seen in the human retina and thought to be a functioning shunt comprised of primitive vascular channels ( $A$, arrows) is compared to a wedge-like thickening of retinal tissue in the puppy retina ( $B$, arrow). On fluorescein angiography, these areas in both human and puppy retinas are seen to become dye stained ( $C$ and $D$, respectively), and the characteristic inwardextending wedge with its apex toward the disk is evident in both.

\section{EFFECT OF ASPIRIN ON PROSTAGLANDIN PRODUCTION}

Methods. One litter of six puppies was subjected to the previously described treatment of doses of aspirin in water or water (control) during oxygen administration. Pairs of littermates consisting of one aspirin-treated and one unmedicated puppy were removed from the chamber and studied after the second and fourth days of treatment, respectively, and a third pair was studied 3 days after termination of all treatments and oxygen exposure. Each puppy was anesthetized with sodium pentobarbital, and a femoral artery was retrogradedly catheterized for blood collection. After exsanguination, the thorax was opened, and an aortic segment was excised and placed on ice. (Inasmuch as it was not feasible to collect a large enough volume of retinal blood vessels from a puppy for assay purposes, the possibly unwarrantable 


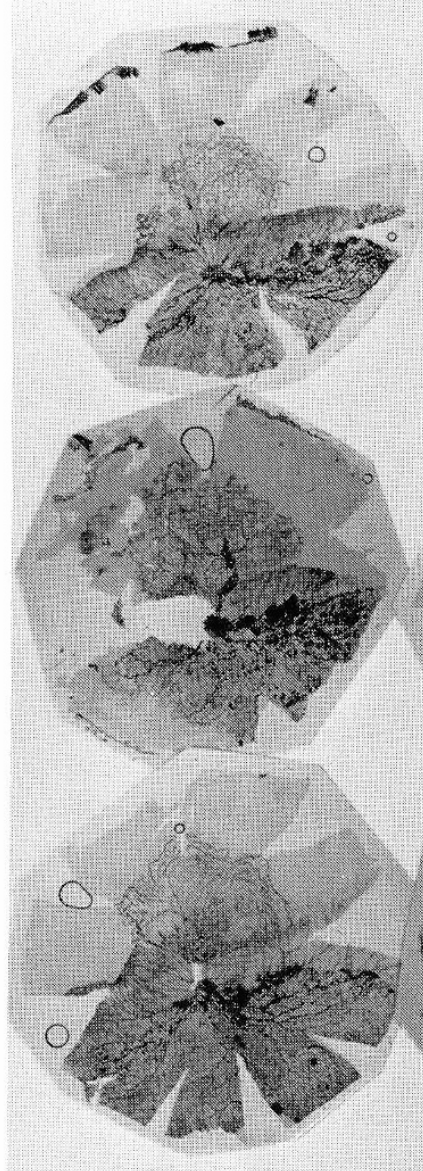

OD

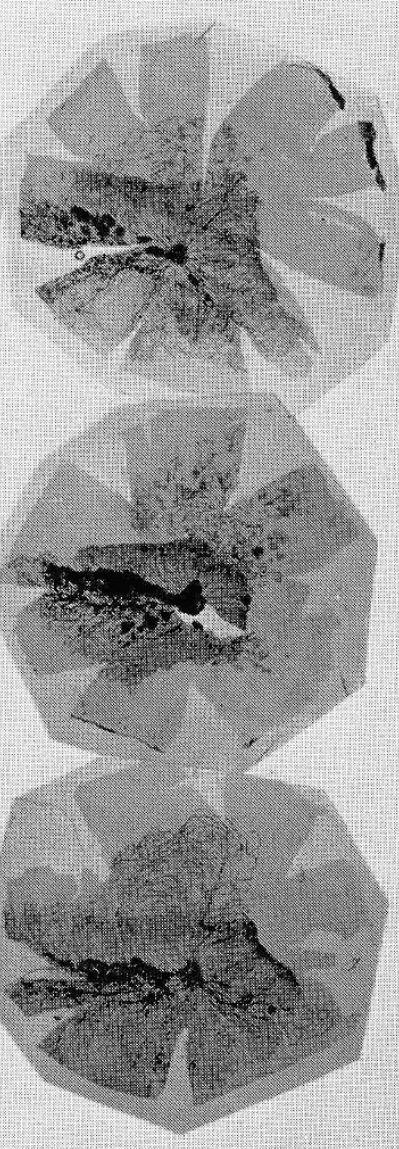

os

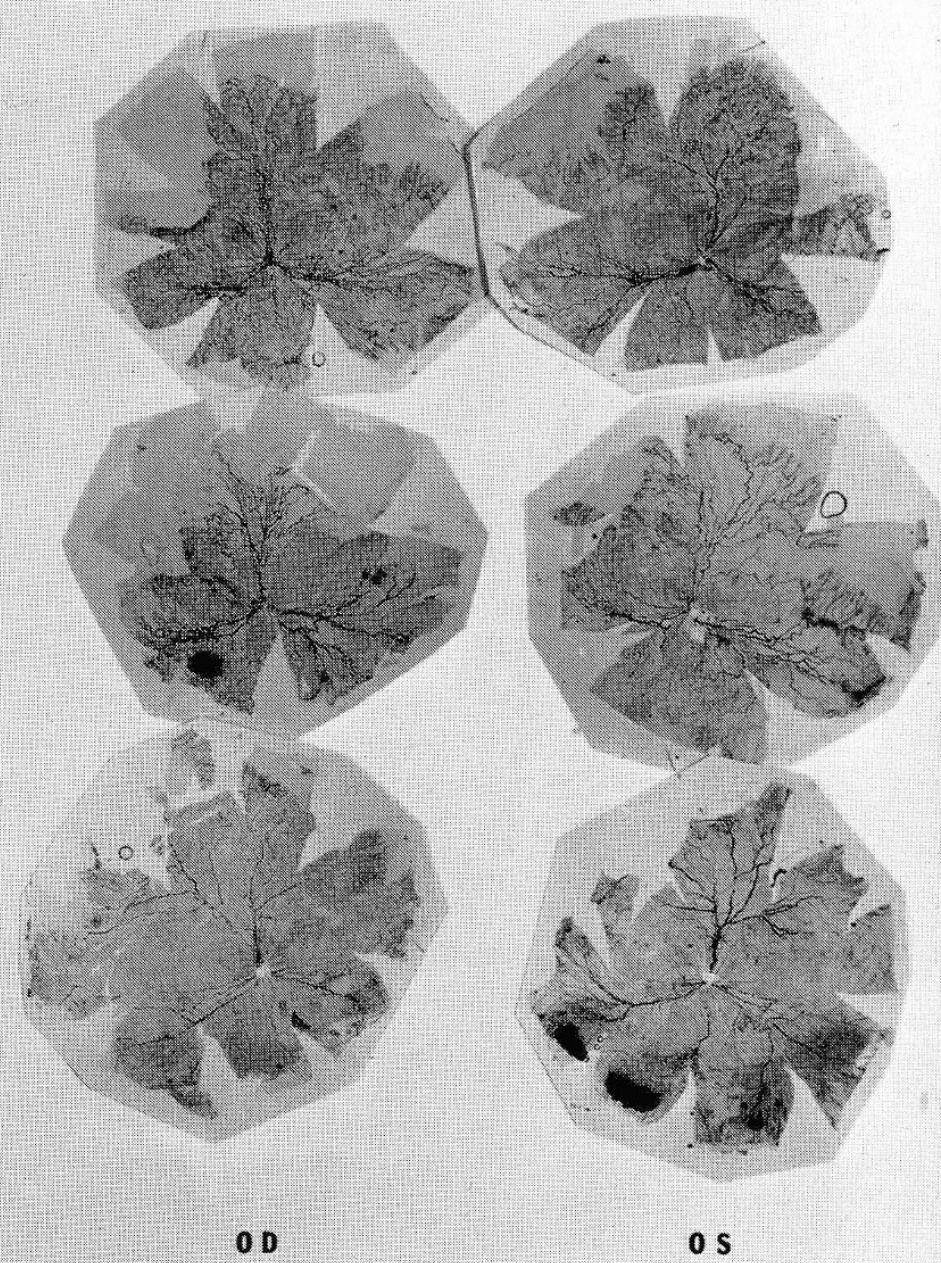

PLACEB O

Fig. 1. Comparison of India ink-filled whole retina flat mounts of both eyes of six beagle puppies from litter 4 (see Table 1). The eyes from a seventh aspirin-treated littermate are not included; although its eyes were not normal, for unexplained reasons, it presented with pathology no more severe than that of the placebo group.

assumption was made that the aorta may be representative of retinal vessels in terms of aspirin effect on prostacyclin production.) Blood samples were mixed in a ratio of 9 to 1 with $3.8 \%$ sodium citrate for preparation of platelet-rich plasma (PRP) (3), and the aortic segments were rinsed with iced $50 \mathrm{mM}$ Tris buffer ( $\mathrm{pH} 7.4)$, blotted, and then cut into rings weighing 3 to $4 \mathrm{mg}$. Twenty-five $\mathrm{mg}$ of aortic rings were placed in $150 \mu \mathrm{l}$ of Tris buffer containing $250 \mu \mathrm{g} / \mathrm{ml}$ of arachidonate and incubated for $1 \mathrm{~min}$ at $37^{\circ} \mathrm{C}$. Aortic production of prostacyclin was assayed by specific radioimmunoassay of its stable metabolite 6-keto- $\mathrm{PGF}_{\mathrm{i} \alpha}$ (22) (coefficient of variation, $11.3 \%$; sensitivity, $3.1 \mathrm{pg}$ ) and confirmed by bioassayed inhibition of ADP-induced aggregation of rabbit platelets (17). Platelet formation of thromboxane was assayed in PRP by $1 \mathrm{~min}$ incubation in the presence of $250 \mu \mathrm{g} / \mathrm{ml}$ of arachidonate. Aliquots of the incubation mixture were radioimmunoassayed for thromboxane $B_{2}$ (13) (coefficient of variation, $13.4 \%$; sensitivity, $5.0 \mathrm{pg}$ ) and confirmed by bioassayable thromboxanelike activity by induction of platelet aggregation in indomethacintreated rabbit PRP (23). Thromboxane and 6-keto- $\mathrm{PGF}_{1 \alpha}$ activity of each aspirin-treated puppy was then compared to those of its control littermates.

Results. Comparisons of production of aortic prostacyclin and platelet thromboxane measured by radioimmunoassay are made in Figure 5 between oxygen-exposed, aspirin-treated, and oxygenexposed, unmedicated puppies. Aspirin-treated puppies were found to have markedly reduced ability to synthesize aortic prostacyclin and platelet thromboxane after 2 and 4 days of treatment (4 and 6 days of age, respectively). The day 4 results show that this dose produced marked inhibition of both pathways. Figure 6 shows similar results obtained by bioassay procedures.

An inhibitory effect of oxygen on production of prostacyclin was also suggested by its decreased formation in aorta from control animals during hyperoxia. Analysis of aortic tissue and platelets from the pair of puppies euthanized 3 days after treatment showed that the aspirin inhibition of prostaglandin biosynthesis was reversing. Salicylate plasma levels ranged from 32 to $45 \mu \mathrm{g} / \mathrm{ml}$ in arterial blood samples obtained $16 \mathrm{hr}$ after the second and fifth daily dosages.

DIRECT VISUALIZATION OF ASPIRIN EFFECTS ON ACUTE RESPONSE OF THE IMMATURE RETINAL VASCULATURE TO HYPEROXIA

Methods. To utilize direct ophthalmoscopic visualization of the retinal vasculature, it was necessary to study slightly older beagle puppies whose ocular media were less hazy: To ensure that the aspirin effect on platelet thromboxane and vessel prostacyclin 


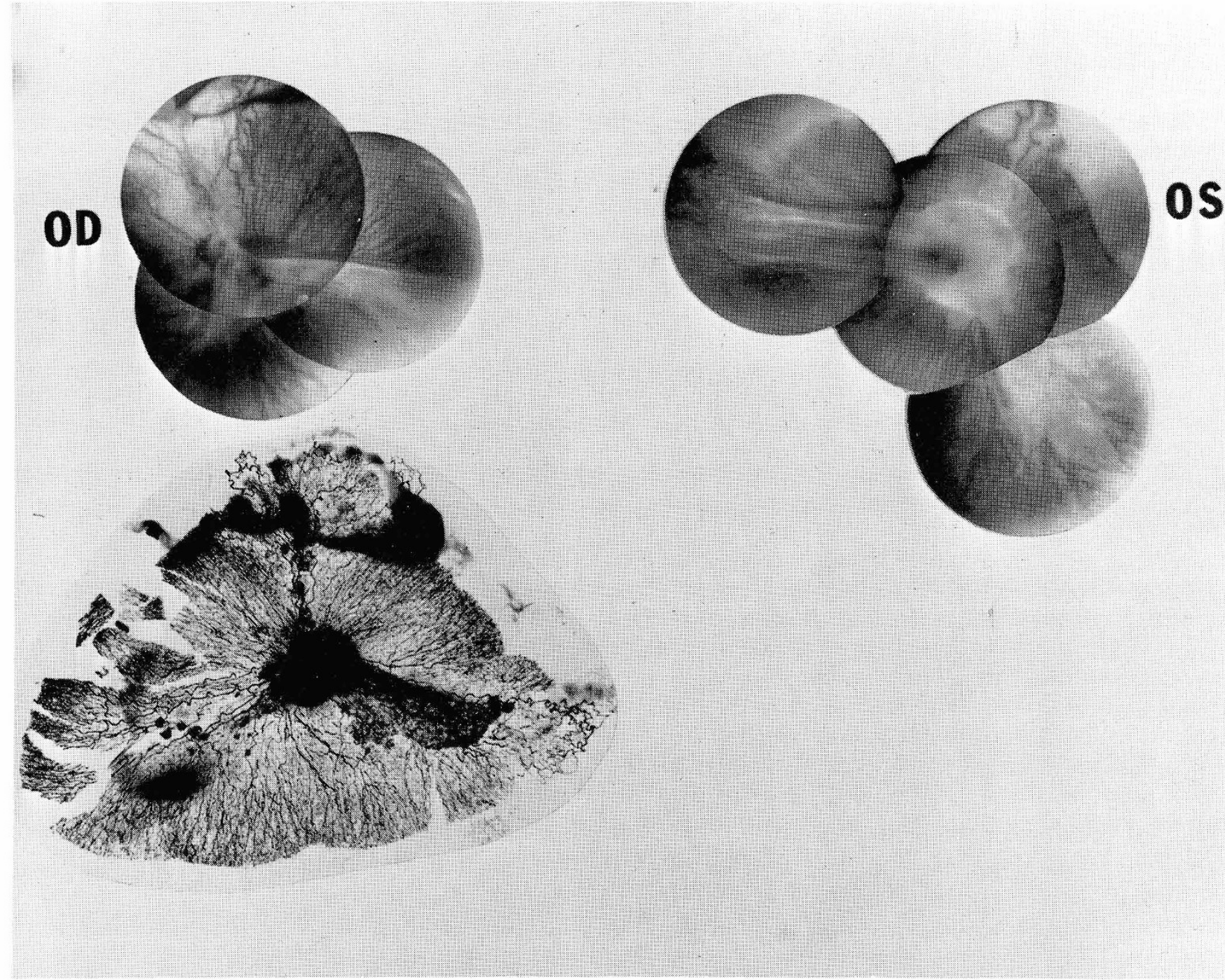

Fig. 2. Composite fundus photographs of the eyes of a beagle puppy showing bilateral grade III cicatricial RLF (falciform retinal folds). The India ink-filled whole retina flat mount of the right eye is also placed in proper orientation beneath its fundus photograph; manipulation of the fellow flat mount resulted in its destruction.

production would be comparable in older puppies, we first determined the dose of aspirin which resulted in cyclo-oxygenase inhibition of an equivalent degree to that previously obtained in 2 - to 4-day-old puppies at a dose of approximately $70 \mathrm{mg} / \mathrm{kg}$. Five 12-day-old puppies (57 days gestation) from a litter of six were weighed and given aspirin at an oral dose of $20,10,5,2$, and 0.2 $\mathrm{mg}$, respectively, and the precise $\mathrm{mg} / \mathrm{kg}$ dose given to each was calculated. [As the starting point, the high aspirin dose administered was determined by an approximation from the human dose shown to inhibit $95 \%$ of platelet thromboxane production (5).] The sixth puppy served as a control. Each was allowed approximately $1.5 \mathrm{hr}$ to absorb the drug, anesthetized with sodium pentobarbital, intubated, and respired on $100 \%$ oxygen for an additional hour. A femoral artery was retrogradely catheterized for blood collection, and bio- and radioimmunoassays were performed on platelets and aortic rings as described above. Based on radioimmunoassay results, it was found that at a dose of $15 \mathrm{mg} /$ $\mathrm{kg}$ in 12-day-old puppies, aspirin produced equivalent inhibitory effects on platelet ( $89 \%$ inhibition) and aortic ( $84 \%$ inhibition) production of cyclooxygenase products as previously observed in 2- to 4-day-old puppies at a dose of $70 \mathrm{mg} / \mathrm{kg}$.

Three litters of 7- to 10-day-old puppies were then used to determine the effects of aspirin on the response of immature retinal vessels to hyperoxia by direct visualization. Each litter was divided into three pairs in such a way that both puppies in each pair were as close as possible to the same body weight. One puppy of each pair was given $15 \mathrm{mg} / \mathrm{kg}$ aspirin suspended in $1 \mathrm{ml}$ of water per os, and the other was given $1 \mathrm{ml}$ of water. One and onehalf $\mathrm{hr}$ after receiving its dose, each puppy was anesthetized with sodium pentobarbital, intubated, and respirated on humidified room air at $38^{\circ} \mathrm{C}$. A femoral artery was retrogradedly catheterized for sampling blood gases and monitoring blood pressure, and the puppy, lying on a heating pad, was positioned with its dilated eye in front of a fundus camera. Blood gases and blood pressure were stabilized for approximately $20 \mathrm{~min}$ (mean values: $\mathrm{P}_{2}=92$ torr; $\mathrm{PCO}_{2}=31$ torr, blood pressure $=66$ torr), and baseline photographs of the retinal blood vessels were made. One-hundred percent oxygen was then substituted for room air, and blood gases and blood pressure were stabilized and monitored (mean values: $\mathrm{PO}_{2}=497$ torr; $\mathrm{PCO}_{2}=34$ torr; blood pressure $=89$ torr). Taking into consideration all the puppies used in these experiments, there were significant variations in blood pressure and blood gas levels, but these levels were nearly identical for each of the weightmatched puppies in a pair.

Observations and photographs were made over equal periods for each puppy in a pair; the observation periods ranged from 75 to $120 \mathrm{~min}$. At the end of each period of observation, India ink was infused via the arterial catheter $(14 \mathrm{mg} / \mathrm{kg}$ body weight at a 

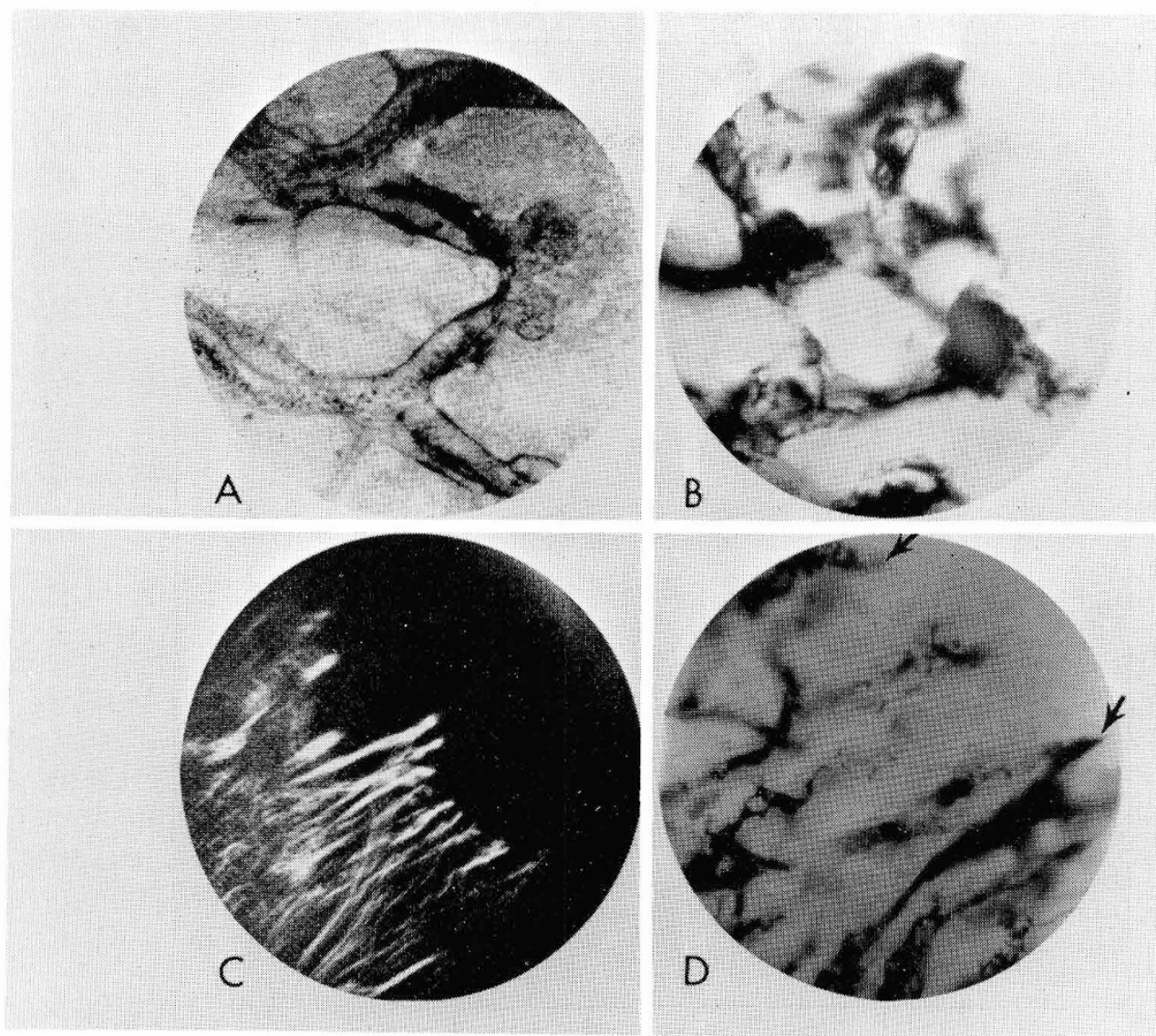

Fig. 3. Comparison of retinal vascular anomalies occurring in human $\operatorname{RLF}(A$ and $C$ ) to those occurring in the beagle puppy model ( $B$ and $D$ ). See text for description. $A$ and $C$ are from material published in B. J. Kushner, D. Essner, I. J. Cohen, J. T. Flynn: Retrolental fibroplasia. II. Pathologic correlation. Archives of Ophthalmology, 95: 29-38 (Copyright 1977, American Medical Association), and J.T. Flynn, G. E. O'Grady, T. Herrera, B. T. Kushner, S. Cantolino, and W. Milam: Retrolental fibroplasia. I. Clinical observations. Archives of Ophthalmology, 95: 217-223 (Copyright 1977, American Medical Association). Reproduced by permission.

rate of $1.9 \mathrm{ml} / \mathrm{min}$ ). The ink was allowed to circulate for $30 \mathrm{sec}$ after completion of infusion with the puppy still breathing $100 \%$ oxygen, and then the puppy was euthanized with a $1.5 \mathrm{ml}$ arterial injection of Somlethol (sodium pentobarbital, $6 \mathrm{~g} / \mathrm{ml}$ ). Retinal flat mounts were then made for evaluation.

Results. The direct observations made on littermate pairs were subsequently confirmed by sequential color and red-free fundus photographs. As noted in previous experiments (7) after the onset of $100 \%$ oxygen breathing, the initial retinal vessel response to hyperoxia varied from eye to eye, even between weight-matched littermates with nearly identical blood pressure and blood gas levels. In most puppies, an initial vasoconstriction followed by dilation occurred during the first $30 \mathrm{~min}$ after onset of oxygen breathing, but in the others, this initial response was absent. After this initial 30-min period, the more gradual secondary constriction previously reported in kittens (7) was observed in the unmedicated puppies, but in the aspirin-treated puppies, vasodilation or return to initial air breathing caliber occurred instead.

Changes in retinal vessel caliber were most obvious in the veins which were more easily seen than arteries through the still hazy media of the young eyes, but major arteries also responded. The first and last fundus photographs from the sequences made of one pair of littermates are shown in Figure 7 . After $2 \mathrm{hr}$ of $100 \%$ oxygen breathing, the aspirin-treated puppy showed significant vessel dilation compared to air breathing vessel caliber, whereas the unmedicated littermate showed significant constriction; both had comparable $\mathrm{PO}_{2}$ and $\mathrm{PCO}_{2}$ levels throughout. To a lesser or greater extent, this same relationship existed between eight of the nine pairs of puppies observed; deterioration of vital signs in one of the ninth pair lead to termination of that experiment.
Left-eye-to-left-eye and right-eye-to-right-eye comparisons of India ink-filled retinal flat mounts from the pairs of littermates were made grossly and microscopically to assess differences in amounts of ink particles in the retinal vessels. Retinas from these eyes enucleated shortly after the first wk of life, however, lacked the structural integrity of those from eyes enucleated after the fourth wk of life, and approximately $25 \%$ were removed in pieces instead of whole. Pigment epithelium also remained attached to many retinas, especially in the periphery. In these latter cases, differentiation between ink particles and pigment epithelium was made possible by the short depth of focus inherent in microscopic examination; the deeper-lying pigment particles were simply not in focus.

From the eight pairs of littermates observed, only 12 pairs of retinas were suitable for comparison. Although comparison of the retinas was somewhat more subjective than the fundus photographs, the findings of four observers were consistent. They found more ink particles in the aspirin-treated puppy of each pair than in the untreated littermates for all but one case, and they were consistent in selecting that case.

The retinal flat mounts from the right eyes of one pair of littermates are shown in Figure 8. There is an obviously greater density of ink particles in that from the aspirin-treated puppy as compared to its unmedicated littermate, confirming the direct observation that the vessels of the aspirin-treated puppy remained open during oxygen breathing. In those few retinas which remained sufficiently intact so that proper orientation could be determined by comparison to fundus photographs, the highest density of ink particles was found in the inferior retinal vasculature (see Figure 8 ). This could not have been due to a settling of 

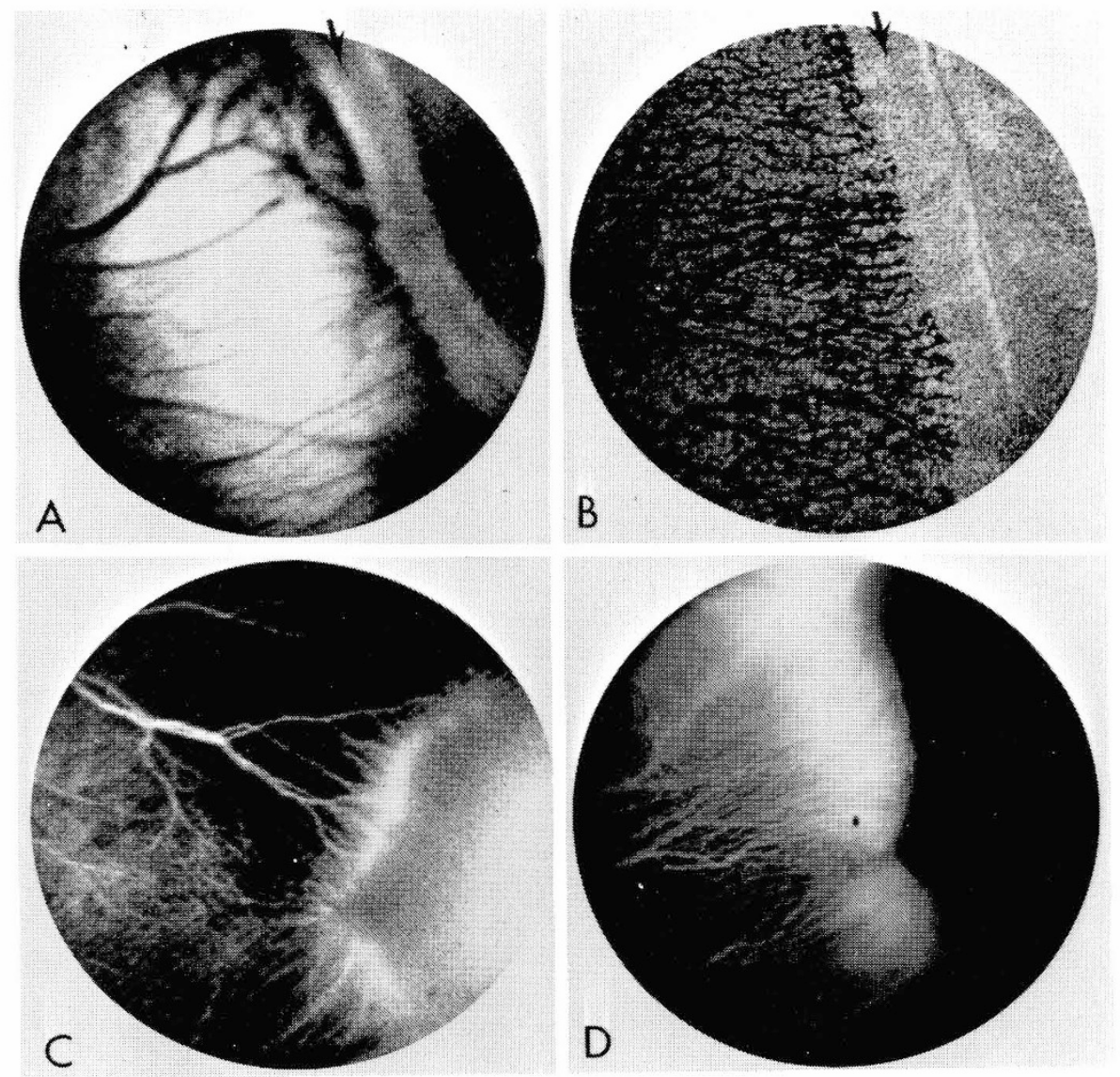

Fig. 4. Comparison of retinal vascular anomalies occurring in human $\operatorname{RLF}(A$ and $C$ ) to those occurring in the beagle puppy model $(B$ and $D$ ). See text for description. $A$ and $C$ are from material published in J. T. Flynn, G. E. O'Grady, T. Herrera, B. J. Kushner, S. Cantolino, and W. Milam: Retrolental fibroplasia. I. Clinical Observations. Archives of Ophthalmology, 95: 217-223 (Copyright 1977, American Medical Association). Reproduced by permission.

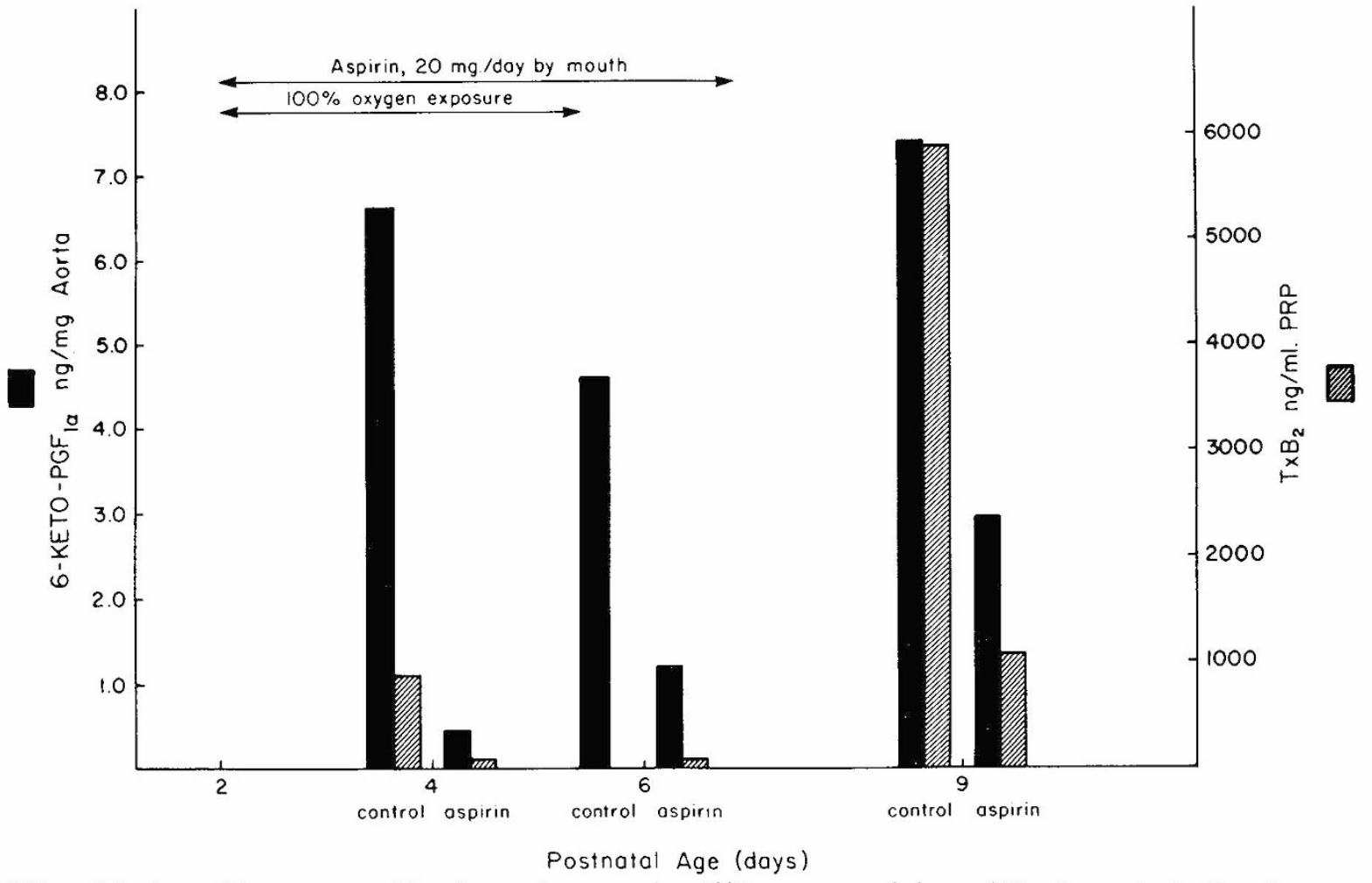

Fig. 5. Effect of in vivo aspirin treatment of beagle puppies exposed to $100 \%$ oxygen on their capability for synthesis of aortic prostacyclin and platelet thromboxane measured in vitro by radioimmunoassay of 6-keto-PGF ${ }_{1 a}$ and thromboxane $\mathrm{B}_{2}$ (see "Methods and Results"). Bars, values obtained by quadruplicate analysis of tissues from individual littermates. The day 6 control dogs' platelets were not assayable because of extensive hemolysis. 


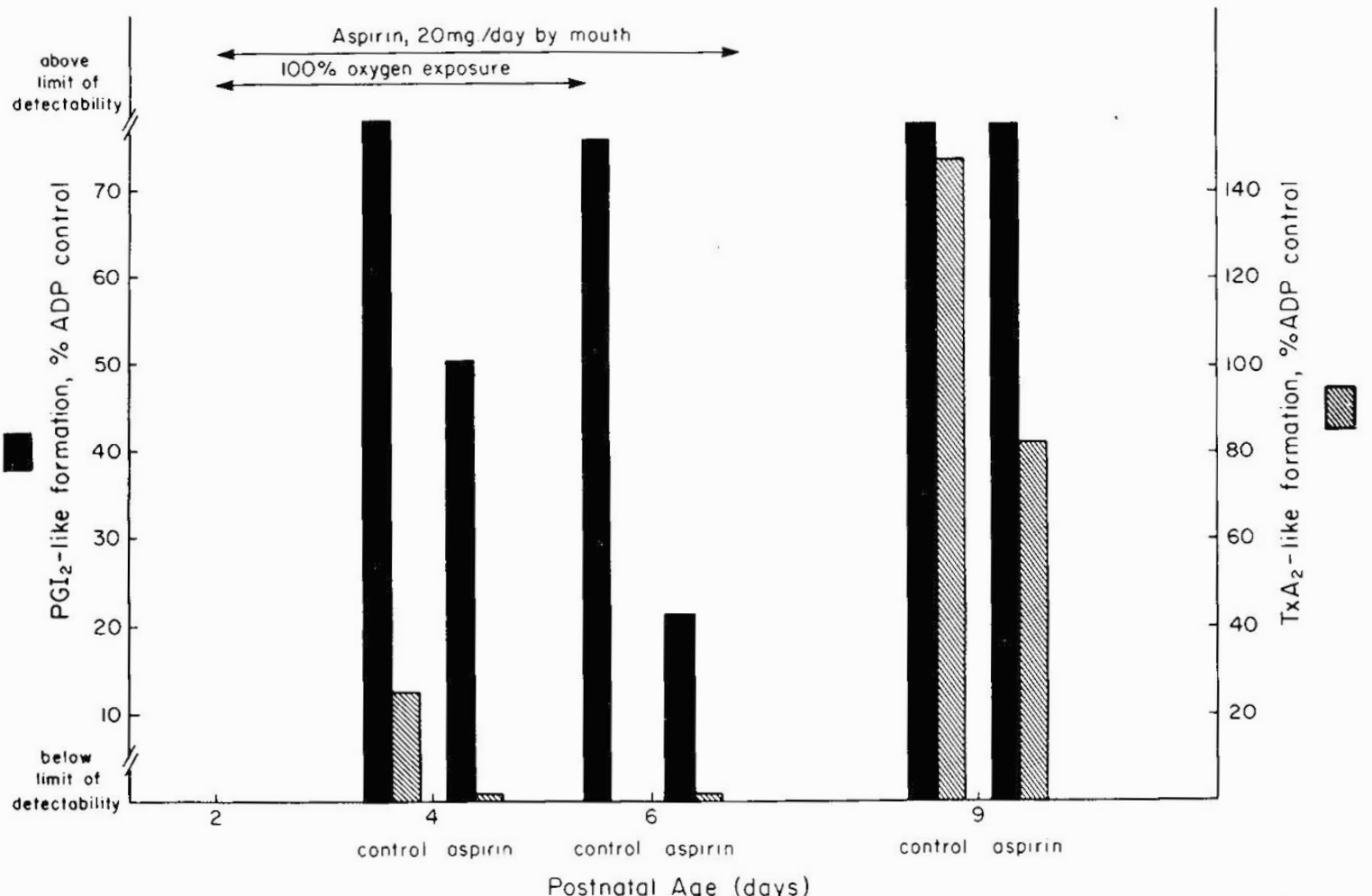

Fig. 6. Effect of in vivo aspirin treatment of beagle puppies exposed to $100 \%$ oxygen on their capability for synthesis of aortic prostacyclin and platelet thromboxane measured in vitro by bioassay of prostacyclin-like activity and thromboxane-like activity (see "Methods and Results"). Bars, values obtained by quadruplicate analysis of tissues from individual littermates. The day 6 control dogs' platelets were not assayable because of extensive hemolysis.

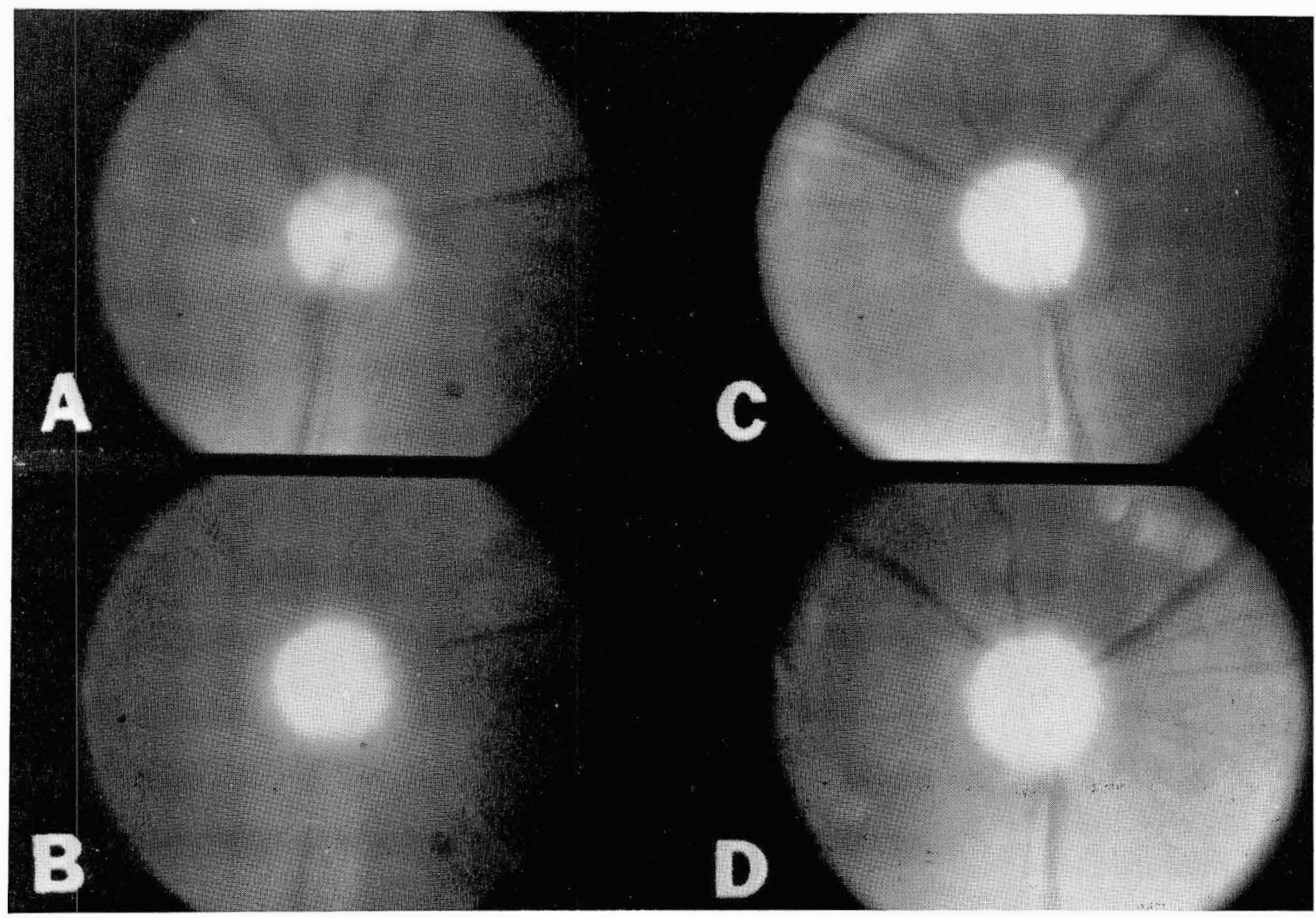

Fig. 7. Comparison of fundus photographs from a pair of beagle puppy littermates. The puppy on the left ( $A$ and $B$ ) was unmedicated, and the puppy on the right $(C$ and $D$ ) was aspirin treated. The top photographs were taken while both animals were breathing ambient air, and those on the bottom were taken after $2 \mathrm{hr}$ of breathing $100 \%$ oxygen. 
particles because all puppies remained on their backs throughout the experiments.

\section{DISCUSSION}

Aspirin administration to puppies was envisioned as a way of testing our hypothesis that immature retinal vasomotion, like vasomotion of the ductus arteriosus, might be mediated by prostaglandins. It is possible, for example, that the local retinal vasoconstriction associated with excessive blood oxygen in RLF results from platelet-derived thromboxane. Aspirin has been shown to inhibit prostaglandin synthesis (25) and to irreversibly inhibit platelet thromboxane production (8). Results of our experiments clearly indicate that oxygen-exposed puppies administered an aspirin dose (approximately $70 \mathrm{mg} / \mathrm{kg}$ ) producing plasma levels within the human therapeutic range developed significantly more severe retinopathy than did their oxygen-exposed, unmedicated littermates. On the other hand, no retinopathy was produced in puppies who received the same aspirin dose regimen but were not exposed to oxygen, indicating that aspirin alone apparently produced no irreversible untoward effects on the immature retinal vessels.

The other experiments reported here were performed in an attempt to elucidate the specific mechanism by which aspirin treatment intensified oxygen-induced retinopathy, presumably by altering the normal in vivo prostaglandin cascade. Unlike the scoring of severity of retinopathy, these results did not lend themselves to statistical analysis; however, the conclusions drawn from them are nonetheless compelling.

Visual confirmation of retinal vasoconstriction in aspirintreated puppies was not possible during the $72 \mathrm{hr}$ of continuous oxygen administration; dense corneal haze and the still patent tunica vasculosa lentis made observation of the fundus by ophthal- moscopy impossible in the intact eyes of the 2- to 5-day-old puppies used in those experiments. Techniques io overcome this problem would have required anesthesia and surgical manipulation of the eye $(1,9)$ and would, therefore, have terminated further usefulness of the puppies in the primary long-term experiments. Use of such techniques, even in other litters of 2- to 5-day-old puppies, was rejected because changes in intraocular pressure relationships would have been produced, with unpredictable vascular effects. Alternatively, litters of puppies ranging in age from 7 to 10 days (the earliest that the fundus can be reasonably visualized by ophthalmoscopy) were used instead to demonstrate that aspirin administration inhibits vasoconstriction of immature retinal vessels during oxygen breathing.

Results of our biochemical studies clearly indicate that aspirin administration at the $70 \mathrm{mg} / \mathrm{kg}$ dose level given the puppies chronically exposed to oxygen causes marked inhibition of both vascular prostacyclin and platelet thromboxane production in beagle puppies (Figs. 5 and 6). Inasmuch as the relative potencies of thromboxane as a vasoconstrictor and prostacyclin as a vasodilator are not known, it is not possible to predict the net vasomotor effect of aspirin inhibition of their formation by platelets and vessel wall, respectively. Moreover, there is no certainty that the in vitro measured effect of in vivo treatment with aspirin really reflects the in vivo situation. Nevertheless, we were able to demonstrate marked inhibition of both platelet thromboxane and aortic prostacyclin production also in 7- to 10-day-old puppies by administration of a $15 \mathrm{mg} / \mathrm{kg}$ aspirin dose; this is the same aspirin dose used in experiments when conversion of the oxygen-induced retinal vasoconstriction in unmedicated puppies into vasodilation in their aspirin-treated littermates was visually observed. This result might imply that the in vivo aspirin effect, if mediated solely by altering prostaglandin biosynthesis, was toward a net vasodilator (prostacyclin) predominance.

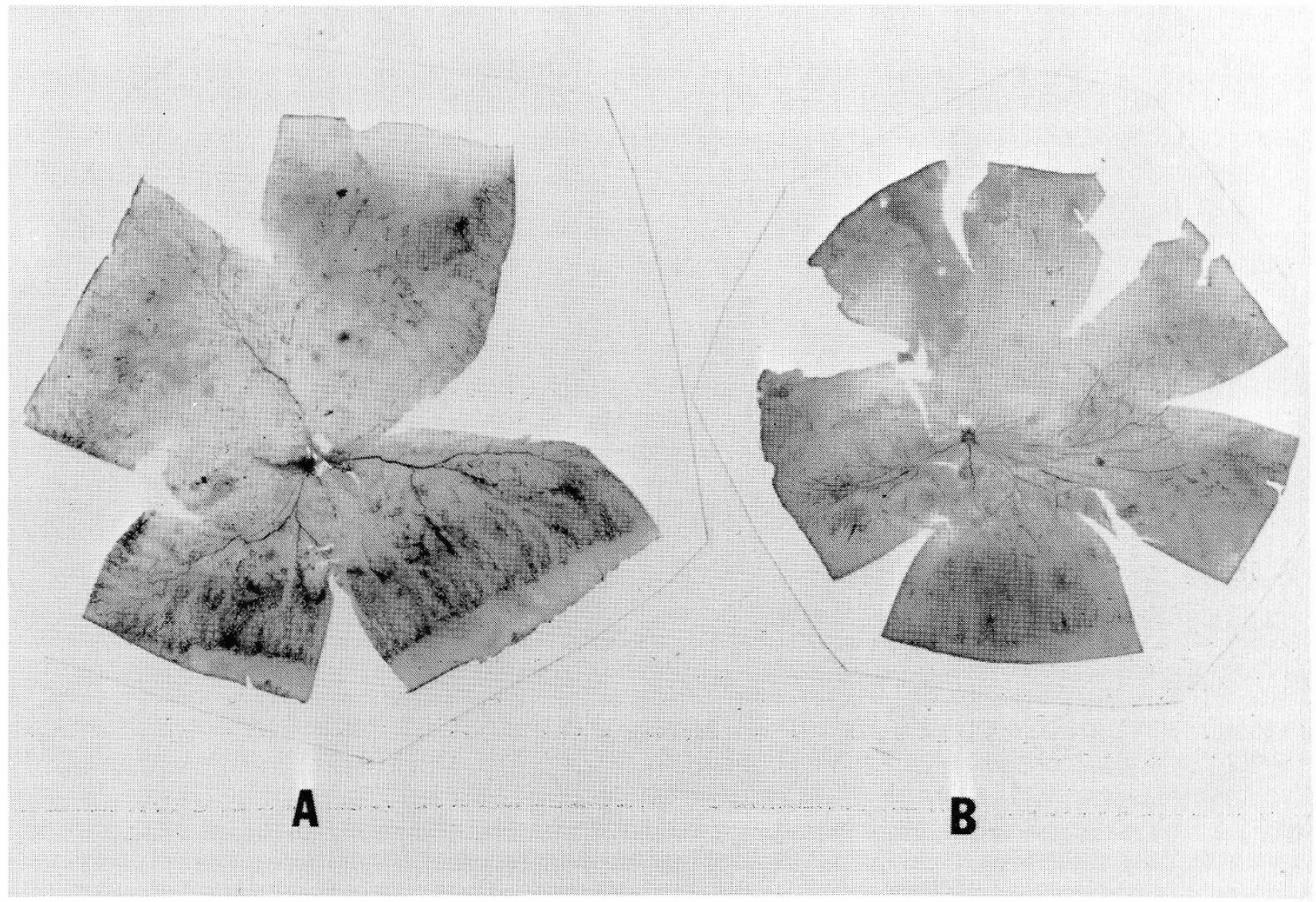

Fig. 8. India ink-filled whole retina flat mounts from the right eyes of a pair of beagle puppy littermates made after $2 \mathrm{hr}$ of breathing $100 \%$ oxygen. Puppy $A$ was aspirin-treated $1 \frac{1 / 2}{\mathrm{hr}}$ before starting oxygen breathing, and puppy $B$ was unmedicated. 
Supported by this result, the visual observations made in the acutely oxygenated, aspirin-treated, and unmedicated littermates lead us to conclude that the more severe retinopathy developed by the chronically oxygenated, aspirin-treated puppies resulted from prostaglandin-induced inhibition of retinal vasoconstriction. This suggests that retinal vasoconstriction may be a normal physiologic mechanism to protect the immature retina from high oxygen tensions. That is, retinal vasoconstriction may be a protective rather than a pathologic process in response to hyperoxia. The implication made here that vasoconstriction is a physiologic property of the perinatal retinal vasculature is supported by the recent fetal and neonatal lamb retinal blood flow data of Peeters et al. (19).

We speculate that the apparently strong vasoconstriction response normally present in both the puppy and kitten may account for the fact that the cicatricial, or endstage, forms of RLF were never produced until vasoconstriction was inhibited by aspirin administration. In our view, the vasoconstriction observed in the premature infant may be only an extreme of a normal physiologic response by which retinal blood flow is modulated during the period when in utero development of the retinal vasculature causes a shift away from total dependence on the adjacent choroidal and hyaloid blood flows for retinal tissue maintenance.

During the early stages of gestation, the mammalian retina is avascular (up to $16 \mathrm{wk}$ in the human fetus). The retinal vessels evolve from a primitive endothelial-lined capillary network laid down in the wake of a wave of mesenchymal tissue which moves radially away from the optic nerve. The mechanism of vasomotion might exist to modulate blood flow while a viable retinal circulation develops. Regulation of blood flow could conceivably protect developing new vessels from too great transmural pressures while they are still structurally incomplete, and it could also help stabilize tissue $\mathrm{PO}_{2}, \mathrm{PCO}_{2}$, or even $\mathrm{pH}$ during the period of transition away from total dependence on the adjacent choroidal and hyaloid bloodflows while the retinal circulation evolves.

It is naive to ignore glibly that "normoxia" in a premature infant is quite abnormal in comparison to the intrauterine oxygen tension. In utero, the immature human retina receives a venouslike blood flow $\left(\mathrm{PO}_{2}=25\right.$ torr; $\mathrm{PCO}_{2}=45$ torr $)$ at a low arterial blood pressure ( 25 to 30 torr), but upon arterialization at birth, blood oxygen tension rises $\left(\mathrm{PO}_{2}=70\right.$ torr; $\mathrm{PCO}_{2}=35$ torr $)$ along with blood pressure (35 to 40 torr). It is possible that constant retinal blood flow during the perinatal period [as reported in the lamb (19), for example] results from some degree of vasoconstriction which occurs at birth concomitantly with rise in blood pressure and thereby establishes that level of retinal vasotonia recognized as clinically normal. It is thus possible that susceptibility of an eye to oxygen-associated retinopathy at birth depends upon the extent to which the vasoconstriction protective response is functional as well as upon the degree of retinal "maturity" attained.

By way of the following scenario, this possibility is consistent with the suggestion of Ashton and Pedler (2) that retinal vessel damage may result from cytotoxic effects of oxygen on endothelial cells. During oxygen breathing, the dilated retinal blood vessels of the aspirin-treated puppies would allow a larger than normal volume of blood to flow through immature vessels, those farthest from the optic nerve. Those vessel walls would be in contact with oxygen radicals (probably derived from dissolved molecular oxygen) which are known to damage cell membranes (14). (In previously reported kitten experiments (7), vasoconstriction was observed only with arterial $\mathrm{PO}_{2}>190$ torr. One hundred percent hemoglobin saturation occurred at about $\mathrm{PO}_{2}=100$ torr; therefore, dissolved molecular oxygen was abundant when vasoconstriction occurred.) Moreover, it may be argued also that "spontaneous" RLF reported in premature infants never administred oxygen (11) or in full-term infants who were administered oxygen (4) might simply be examples of inadequate retinal vasotonia for protection of structurally immature retinal vessels at birth when arterial blood oxygen tension and blood pressure suddenly rise. Thus, our present interpretation of the immature retinal vascular response can potentially explain virtually all the observations reported on newborn animal eyes as well as those made clinically on human infants.

At the same time, the possibility should not be ignored that due to inhibited vasoconstriction, the immature vessels may also have experienced inordinately high transmural pressures, accounting for the hemorrhages we observed. The fact that less severe retinopathy was produced in littermates where vasoconstriction was uninhibited might then be attributable to less or no contact between immature vessel walls and high oxygen content blood as well as to presence of much lower or normal transmural pressures. In any case, occurrence of a presumed normal degree of vasoconstriction at birth would add resistance to blood flow throughout the retinal vasculature in such a way that the most peripheral, and hence the most structurally immature, vessels would experience the smallest increase in transmural pressure when arterial blood pressure increases significantly. Thus, the possibility arises that factors other than oxygen alone may contribute to genesis of RLF

The unexpected production of grade III cicatricial retinopathy (falciform retinal fold) in several of the most severely affected aspirin-treated, oxygen-exposed puppies is by itself a significant consequence of our experiments. Inasmuch as the usefulness and reliability of an animal model depends primarily upon the extent to which it mimics the human disease, production of cicatricial RLF in the puppy eye answers a major criticism of the RLF animal model and thereby strengthens the confidence with which results from experimental animal studies might be extrapolated to the clinical situation. It would, nevertheless, be premature at this time to interpret our results as indicating possible untoward effects of aspirin-like drugs on the human fetus because the data in young puppies may not be directly applicable to human infants. Inasmuch as aspirin-like drugs undergo placental transport (16), these preliminary animal data, if confirmed independently by other investigators, should alert investigators using prostaglandin synthesis inhibitor to arrest labor or close the patent ductus to examine and possibly test this concept clinically.

\section{REFERENCES AND NOTES}

1. Ashton, N., and Cook, C.: Direct observation of the effect of oxygen on developing vessels: Preliminary report. Br.J. Ophthalmol., 38: 433 (1954)

2. Ashton, N., and Pedler, C.: Studies on developing retinal vessels. IX. Reaction of endothelial cells to oxygen. Br. J. Ophthalmol., 46: 257 (1962).

3. Born, G. V. R.: Aggregation of blood platelets by adenosine diphosphate and its reversal. Nature (Lond.), 194: 927 (1962).

4. Brockhurst, R. J., and Chisti, M. I.: Cicatricial retrolental fibroplasia: Its occurrence without oxygen administration and in full term infants. Albrecht von Graefes Arch. Klin. Exp. Ophthalmol., 195: 113 (1975).

5. Burch, J. W., Stanford, N., and Majerus, P. W.: Inhibition of platelet prostaglandin synthetase by oral aspirin. J. Clin. Invest., 61: 314 (1978).

6. Clyman, R. I., Mauray, F., Heymann, M. A., and Rudolph, A. M.: Ductus arteriosus: developmental response to oxygen and indomethacin. Prostaglandins, 15: 993 (1978)

7. Flower, R. W., and Patz, A.: Oxygen studies in retrolental fibroplasia: IX. The effects of elevated arterial oxygen tension on retinal vascular dynamics in the kitten. Arch. Ophthalmol., 85: 197 (1971).

8. Flower, R. J.: Drugs which inhibit prostaglandin biosynthesis. Pharmacol. Rev. 26: 33 (1974).

9. Flower, R. W., Patz, A., and Speiser, P.: New method for studying immature retinal vessels in vivo. Invest. Ophthalmol., 7: 366 (1968)

10. Flynn, J. T., O'Grady. G. E., Herrera, J., Kushner, B. J., Cantolino, S., and Milam, W.: Retrolental fibroplasia: I. Clinical observations. Arch. Ophthalmol. 95: 217 (1977).

11. Foos, R. Y.: Acute retrolental fibroplasia. Albrecht von Graefes Arch. Klin. Exp. Ophthalmol, 195: 87 (1975).

12. Friedman, W. F., Hirschklau, M. J., Printz, M. P., Pitlick, P. T., and Kirkpatrick, S. E.: Pharmacologic closure of patent ductus arteriosus in the premature infant. N. Engl. J. Med., 295: 526 (1976).

13. Granstrom, E., Kindahl, H., and Samuelsson. B.: Radioimmunoassay for throm boxane B. Anal. Lett., 9:611 (1976).

14. Harman, D., and Piette, L. H.: Free radical theory of aging: Free radical reactions in serum. J. Gerontol., 21: 560 (1966)

15. Kushner, B. J., Essner, D., Cohen, I. J., and Flynn, J. T.: Retrolental fibroplasia: II. Pathologic correlation. Arch. Ophthalmol., 95: 29 (1977).

16. Levy. G., and Garrettson, L. K.: Kinetics of salicylate elimination by newborn 
infants of mothers who ingested aspirin before delivery. Pediatrics, 53: 201 (1974).

17. Moncada, S., Higgs. E. A.. and Vane, J. R.: Human arterial and venous tissues generate prostacyclin (Prostaglandin $\mathrm{X}$ ), a potent inhibitor of platelet aggregation. Lancet, 1 : 18 (1977)

18. Patz, A.. Hoeck. L. E., and deLa Cruz. E.: Studies on the effect of high oxygen administration in retrolental fibroplasia: nursery observations. Am. J. Ophthalmol., 35: 1248 (1952).

19. Peeters, L. L. H.. Sheldon, R. E.. Jones, M. P.. Jr., and Battaglia. F. C.: Retinal and choroidal blood flow in unstressed fetal and newborn lambs. Pediatr. Res., 14: 1047 (1980).

20. Phelps. D. L.. and Rosenbaum. A. L.: The role of tocopherol in oxygen-induced retinopathy. Pediatrics, 59:998 (1977).

21. Roth. G. J., and Majerus. P. W.: The mechanism of the effect of aspirin on human platelets. J. Clin. Invest.. 56: 624 (1975).

22. Salmon, J. A.: A radioimmunoassay for 6-keto-prostaglandin $F_{1 \alpha}$. Prostaglandins, 15: 383 (1978).

23. Silver, M. J.. Smith, J. B., Ingerman, C., and Kocsis, J. J.: Arachidonic acid

Copyright $(C) 1981$ International Pediatric Research Foundation. Inc. $0031-3998 / 81 / 1509-1293 \$ 02.00 / 0$ induced human platelet aggregation and prostaglandin production. Prostaglandins, 4: 863 (1973).

24. Terry. T. L.: Extreme prematurity and fibroblastic overgrowth of persistent vascular sheath behind each crystalline lens: I. Preliminary report. Am. J. Ophthalmol., 25: 203 (1942).

25. Villa, S., and Gaetano, G.: Prostacyclin activity in rat vascular tissues. Fast, long lasting inhibition by treatment with lysine acetylsalicylate. Prostaglandins, 14 : 1117 (1977).

26. The authors gratefully acknowledge the helpful suggestions and criticisms of Drs. A. Patz, G. A. Gole, and M. D. Jones, Jr. We also thank Dr. E. D. Mellits who assisted in the statistical evaluation of our data.

27. Requests for reprints should be addressed to: Robert W. Flower, The Johns Hopkins University, Applied Physics Laboratory, Johns Hopkins Road, Laurel MD 20810 (USA).

28. This research was supported in part by NIH grants EY-02482 and EY-00205

29. Received for publication October 3, 1980.

30. Accepted for publication January 2,1981 . 